Artículo

\title{
Desarrollo de un método eficiente para la micropropagación de orégano
}

\author{
Reyna Rojas-García ${ }^{1}$ \\ Francisca Ramírez-Godina ${ }^{1}$ \\ Barbarita Companioni-González ${ }^{1}$ \\ Ileana Vera-Reyes ${ }^{2}$ \\ Valentín Robledo-Torres ${ }^{1}$ \\ Hermila Trinidad García-Osuna ${ }^{1 \S}$ \\ ${ }^{1}$ Universidad Autónoma Agraria Antonio Narro. Calzada Antonio Narro núm. 1923, Saltillo, Coahuila, \\ México. CP. 25350. Tel. 844 4110287. (sunrey_rg@ hotmail.com; godramf@gmail.com; \\ bcompanioni2007@gmail.com; robledo3031@gmail.com). ${ }^{2}$ Centro de Investigación en Química Aplicada. \\ Enrique Reyna H. núm. 140, San José de los Cerritos, Saltillo, Coahuila, México. CP. 25294. Tel. 844 \\ 4389830. (ileana.vera@ciqa.edu.mx). \\ ${ }^{\S}$ Autor para correspondencia: hgosuna@ hotmail.com.
}

\section{Resumen}

Lippia graveolens u orégano mexicano, es una planta de importancia económica y la recolección del follaje se realiza principalmente de poblaciones naturales. La micropropagación propone una alternativa para la producción de material, con alta calidad fitosanitaria y propagación en menor tiempo de individuos seleccionados por sus características de valor comercial. El objetivo de este trabajo fue establecer un protocolo para la propagación in vitro de Lippia graveolens. La investigación se realizó en el año 2018-2019. El establecimiento se logró con segmentos nodales en medio basal MS, en esta etapa se recuperó $57.32 \%$ de explantes axénicos. Para la etapa de propagación se adicionó al medio MS cuatro concentraciones $\left(0,1,2\right.$ y $\left.3 \mathrm{mg} \mathrm{L}^{-1}\right)$ de bencilaminopurina (6-BAP), se evaluó número de brotes y altura de plántula, los mejores resultados en la multiplicación se lograron con $3 \mathrm{mg} \mathrm{L}^{-1}$ de 6-BAP, obteniéndose 40.35 brotes por explante. La longitud de brote fue mayor en los tratamientos sin reguladores de crecimiento. Para el enraizamiento, se ensayó con tres concentraciones $\left(0,0.1,0.3\right.$ y $\left.0.5 \mathrm{mg} \mathrm{L}^{-1}\right)$ de auxinas (ácido indol acético y ácido indol butírico) y se evaluó el porcentaje de enraizamiento y la longitud de la raíz obteniéndose igual resultado con y sin reguladores auxínicos. En la fase de aclimatación se evaluó el fotoperiodo (16 h y $24 \mathrm{~h}$ ) con y sin micorrizas (Glomus intraradicens). El mejor tratamiento se obtuvo con 24 h luz y con adición de micorrizas con un $86.11 \%$ de supervivencia.

Palabras clave: Glomus intraradicens, biotización, fotoperiodo.

Recibido: marzo de 2021

Aceptado: julio de 2021 


\section{Introducción}

Los recursos forestales no maderables (RFNM) presentan una importancia, ecológica, económica y cultural de las comunidades indígenas y rurales de nuestro país. La colecta de estos recursos puede tener efectos negativos asociados al nivel de abundancia o pérdida de la biodiversidad. Por lo tanto, es necesario la búsqueda de nuevas estrategias de manejo sustentable que permitan minimizar el impacto. Se ha observado, que la producción especializada de RFNM en un sistema agroforestal con mayor compromiso ecológico, favorece resultados ambientales y de subsistencia (Kusters et al., 2006).

El orégano es un RFNM de distribución mundial, el cual está representado principalmente por dos especies: Origanum vulgare (Lamiaceace), nativo de Europa y Lippia graveolens (Verbenaceae), originario de América. La producción global del orégano es estimada en alrededor de $15000 \mathrm{t}$, siendo Turquía el principal productor seguido de México (García-Pérez et al., 2012). L. graveolens conocida como orégano mexicano es una planta aromática nativa del Sureste de EUA, México, Guatemala, Nicaragua y Honduras (Martínez-Rocha et al., 2008) pertenece a la familia Verbenaceae.

Su aprovechamiento implica la remoción de hojas y tallos (Ocampo-Velázquez et al., 2009; Granados-Sánchez et al., 2013). Debido a su alto contenido de aceite esencial, el orégano es frecuentemente usado como sazonador en preparación de comida y como planta medicinal, es usada para tratar dolores de cabeza, reumáticos y como diurético (Laferriere et al., 1991; Marques et al., 2009).

El timol y carvacrol son dos de los compuestos activos del aceite esencial obtenido del orégano mexicano. Estos compuestos son de interés especial por sus propiedades antimicrobianas contra especies de Vibrio, muestra efecto antimicótico contra $F$. oxysporum en semillas de tomate y es acaricida contra Rhipicephalus microplus (Paredes-Aguilar et al., 2007; Cueto-Wong et al., 2010; Martínez-Velázquez et al., 2011) y antioxidantes (Dunford y Vázquez 2005; Ávila-Sosa et al., 2010).

En el estado de Coahuila el aprovechamiento de L. graveolens se realiza en ocho municipios; sin embargo, es en la región sureste del estado, en los municipios de Parras de la Fuente, General Cepeda y Ramos Arizpe, donde se obtiene la mayor producción y se genera anualmente un promedio 700 t de hoja seca (Villavicencio-Gutiérrez et al., 2010). Se extrae principalmente de poblaciones naturales y su aprovechamiento coincide con la floración y altera la formación de frutos y semillas (Ocampo-Velázquez et al., 2009).

Con el paso del tiempo la recolección de plantas puede disminuir el tamaño y densidad poblacional, así como la reducción de la diversidad genética en las zonas de aprovechamiento, por lo tanto, surge la necesidad de optimizar un protocolo de propagación que permita la propagación masiva de individuos de alta calidad fitosanitaria, además de presentar características quimiotípicas deseables, mediante herramientas biotecnológicas.

El primer protocolo de propagación en L. graveolens fue realizado por Castellanos-Hernández et al. (2013) quienes emplearon semilla para generar plantas in vitro de las cuales obtuvieron explantes para la inducción de brotes vía organogénesis directa de yemas axilares ero al provenir 
de semillas presentan alta variabilidad en la concentración de timol y carvacrol, lo cual repercute en su rendimiento y calidad (Soto et al., 2007). El objetivo del presente trabajo fue optimizar un protocolo de propagación in vitro de la especie L. graveolens mediante cultivo de segmentos nodales.

\section{Materiales y métodos}

La presente investigación se realizó en el Laboratorio de Cultivo de Tejidos Vegetales del Departamento de Fitomejoramiento de la Universidad Autónoma Agraria Antonio Narro, en Saltillo Coahuila.

\section{Material vegetal}

Los explantes fueron colectados de plantas sembradas de una plantación comercial de dos años, situada en la localidad de El Amparo (La Peña) municipio de Parras de la Fuente, Coahuila.

\section{Etapa 1. Establecimiento de segmentos nodales de L. graveolens}

Se tomaron explantes de segmentos nodales de las plantas madre a principios de primavera, durante el mes de marzo de 2018. Se realizó un pretratamiento de desinfección de los segmentos para eliminar los contaminantes externos, para esto se mantuvieron durante media hora en una solución de cloro al $10 \%(\mathrm{v} / \mathrm{v})$ a la cual se agregaron cuatro gotas de detergente comercial, después se enjuagaron con agua corriente. En esta etapa el protocolo de asepsia de los explantes fue realizado en la campana de flujo laminar.

El segmento nodal tuvo una altura de 2 a $3 \mathrm{~cm}$ aproximadamente, se le aplicó una solución de etanol al $70 \%$ (v/v) durante un minuto, después se colocaron en una solución de hipoclorito de sodio $\left(\right.$ Cloralex $^{\circledR}$ ) al $20 \%$ durante 20 min y posteriormente fueron enjuagados tres veces con agua destilada estéril y sembrados en tubos de ensaye con $20 \mathrm{ml}$ de medio de cultivo Murashige y Skoog (1962) libre de reguladores y suplementado con $100 \mathrm{mg} \mathrm{L}^{-1}$ de myo-inositol, $1 \mathrm{mg} \mathrm{L}^{-1}$ de tiamina $\mathrm{HCl}, 1 \mathrm{mg} \mathrm{L}^{-1}$ de piridoxina- $\mathrm{HCl}, 50 \mathrm{mg} \mathrm{L}^{-1}$ de cisteína, $30 \mathrm{~g} \mathrm{~L}^{-1}$ de sacarosa, $4 \mathrm{~g} \mathrm{~L}^{-1}$ de phytagel (Sigma Aldrich) ajustando a un $\mathrm{pH}$ de 5.7 y esterilizado en autoclave por $20 \mathrm{~min}$ a $121^{\circ} \mathrm{C}$.

Se colocó un explante por tubo y se establecieron 250 explantes de $2 \mathrm{~cm}$ aproximadamente. Las condiciones de cultivo fueron: temperatura de $25 \pm 1{ }^{\circ} \mathrm{C}$, con $16 \mathrm{~h}$ luz y $8 \mathrm{~h}$ de oscuridad a 2500 lux. Después de 30 días se procedió a la evaluación de las siguientes variables: porcentaje de explantes contaminados, porcentaje de explantes muertos, y porcentaje de explantes sanos.

\section{Etapa II. Multiplicación de L. graveolens}

Para esta etapa se emplearon segmentos nodales y apicales con cuatro hojas obtenidos de la etapa anterior, los cuales fueron colocados en frascos de vidrio de $6 \mathrm{~cm}$ de diámetro y $8 \mathrm{~cm}$ de altura, con $20 \mathrm{ml}$ de medio MS adicionado con cuatro concentraciones de 6-BAP $\left(0,0.1,2\right.$ y $\left.3 \mathrm{mg} \mathrm{L}^{-1}\right)$ suplementado con $100 \mathrm{mg} \mathrm{L}^{-1}$ de myo-inositol, $1 \mathrm{mg} \mathrm{L}^{-1}$ de tiamina $\mathrm{HCl}, 1 \mathrm{mg} \mathrm{L}^{-1}$ de piridoxina$\mathrm{HCl}, 30 \mathrm{~g} \mathrm{~L}^{-1}$ de sacarosa y $4 \mathrm{~g} \mathrm{~L}^{-1}$ de phytagel (Sigma Aldrich) ajustado a un $\mathrm{pH}$ de $5.7 \mathrm{y}$ esterilizado en autoclave por $20 \mathrm{~min}$ a $121^{\circ} \mathrm{C}$. 
Se colocaron cuatro explantes por frasco y se obtuvieron un total de 8 tratamientos, cada uno con cinco repeticiones. Transcurridos 30 días después de la incubación en la cámara de cultivo se procedió a determinar los parámetros: número de brotes/explantes y longitud del brote. Los explantes fueron subcultivados de forma continua en las mismas combinaciones durante cinco subcultivos cada 30 días. En el momento del subcultivo se evaluó en cada uno de los tratamientos, número de brotes/explantes y la longitud del brote.

Los brotes obtenidos en la etapa de multiplicación fueron subcultivados en medio MS adicionado con $0.01 \mathrm{mg} \mathrm{L}^{-1}$ de ácido naftalen acético (ANA), $100 \mathrm{mg} \mathrm{L}^{-1}$ de myo-inositol, $1 \mathrm{mg} \mathrm{L}^{-1}$ de tiamina $\mathrm{HCl}, 1 \mathrm{mg} \mathrm{L}^{-1}$ de piridoxina-HCl, $30 \mathrm{~g} \mathrm{~L}^{-1}$ de sacarosa, $0.025 \mathrm{~g} \mathrm{~L}^{-1}$ de cistena y $4 \mathrm{~g} \mathrm{~L}^{-1}$ de phytagel (Sigma Aldrich) ajustado a un $\mathrm{pH}$ de 5.7 y esterilizado en autoclave por 20 min a $121^{\circ} \mathrm{C}$.

\section{Etapa III. Enraizamiento}

Se tomaron brotes de $2.5 \mathrm{~cm}$ y se colocaron en frascos de vidrio de $6 \mathrm{~cm}$ de diámetro y $8 \mathrm{~cm}$ de altura, con $20 \mathrm{ml}$ de medio MS adicionado con tres concentraciones de ácido indol acético (AIA), ácido indol butírico (AIB) y un testigo. Los tratamientos fueron: IA0.1 $=0.1 \mathrm{mg} \mathrm{L}^{-1} \mathrm{de}$ AIA, IA0.3= $0.3 \mathrm{mg} \mathrm{L}^{-1}$ de AIA, IA0.5 = $0.5 \mathrm{mg} \mathrm{L}^{-1}$ de AIA, IB0.1=0.1 $\mathrm{mg} \mathrm{L}^{-1} \mathrm{de}$ AIB, IB0.3=0.3 mg L $\mathrm{m}^{-1}$ AIB, IB0.5=0.5 $\mathrm{mg} \mathrm{L}^{-1}$ de AIB y AT= testigo).

Se colocaron cuatro explantes por frasco y se obtuvieron un total de seis tratamientos y un testigo absoluto, cada uno con cinco repeticiones y fueron colocados en el cuarto de incubación bajo las condiciones mencionadas en el establecimiento. Transcurridos 30 días se evaluó la longitud de la raíz.

\section{Etapa IV. Aclimatación}

Para esta etapa, se seleccionaron plántulas de $3-3.5 \mathrm{~cm}$ de altura, se establecieron en un diseño completamente al azar con cinco tratamientos: (T1) testigo (con riegos), (T2) fotoperiodo de $16 \mathrm{~h}$ (2 500 lux), (T3) fotoperiodo de 16 h (2 500 lux) más micorrizas; (T4) fotoperiodo de 24 h (2 500 lux) y (T5) fotoperiodo de 24 h (2 500 lux) más micorrizas.

Para el endurecimiento de las plántulas los frascos fueron abiertos una semana antes de su colocación en invernadero, posteriormente las plántulas fueron removidas del medio de cultivo, lavadas con agua destilada y se aplicó micorrizas (Glomus intraradicens 46 esporas $\mathrm{g}^{-1}$ ) a los tratamientos T3 y T5. Cada tratamiento consistió en 36 plántulas colocadas en charolas con sustrato peat-moss:perlita (1:1), el material vegetal se colocó en el invernadero, con equipo humidificador (Hidrofogger) aplicando $1.5 \mathrm{~L} \mathrm{~h}^{-1}$ cada $4 \mathrm{~h}$ y a los 30 días se evaluó el porciento de sobrevivencia.

\section{Análisis estadístico}

Para la etapa de propagación se realizó un diseño completamente al azar con arreglo factorial ( $2 \mathrm{x}$ 4) donde los factores fueron: a) tipo de explantes (segmentos nodales y apicales); y b) concentraciones de 6-BAP $\left(0,1,2\right.$ y $\left.3 \mathrm{mg} \mathrm{L}^{-1}\right)$. En la etapa de enraizamiento se realizó un análisis de varianza (Anova) con diseño completamente al azar y las medias fueron comparadas mediante la prueba de Tukey $(p<0.05)$. Los análisis se realizaron con el lenguaje de computación estadístico $\mathrm{R}$ (Core Team 2017). Para la etapa de aclimatización se evaluó un porcentaje de sobrevivencia. 


\section{Resultados}

\section{Etapa I. Establecimiento de segmentos nodales de L. graveolens}

La concentración y el tiempo de aplicación del desinfectante influyeron de forma significativa en los resultados de este experimento (Cuadro1). Los mejores resultados muestran 57.32\% de sobrevivencia de los explantes al aplicar el método de desinfección (tiempo y concentración) En el testigo se observó $100 \%$ de contaminación y explantes muertos. La contaminación en el tratamiento con hipoclorito de sodio fue de $32.52 \%$, mientras los explantes muertos representaron $10.16 \%$.

Cuadro 1. Efecto del hipoclorito de sodio en la desinfección de explantes nodales en L. graveolens.

$\mathrm{NaClO}$ Tiempo

(\%) (min)

$(\%)$ de explantes contaminados $(\%)$ de explantes muertos $(\%)$ de explantes sanos

\begin{tabular}{ccccc}
\hline $0 \%$ & 20 & $100 \mathrm{~b}$ & 0 & 0 \\
$20 \%$ & 20 & $32.52 \mathrm{a}$ & 10.16 & 57.32
\end{tabular}

Media con letras iguales no difieren estadísticamente (One-Way Anova, Tukey, $p<0.05$ ).

\section{Etapa II. Multiplicación de L. graveolens}

Los brotes obtenidos de las dos yemas adventcias fueron empleados como fuente de explantes en el proceso de multiplicación. La dosis de 6-BAP utilizada en el medio de cultivo, se observó diferencias significativas $(p<0.05)$ en la multiplicación y longitud del brote en ambos tipos de explantes (segmento nodal y apical) de L. graveolens (Cuadro 2).

Cuadro 2. Efecto de diferentes concentraciones de 6-BAP en la multiplicación de brotes de $L$. graveolens en dos tipos de explantes. $\mathrm{B} 0=$ testigo; $\mathrm{B} 1=\mathrm{BAP} 1 \mathrm{mg} \mathrm{L}^{-1} ; \mathrm{B} 2=\mathrm{BAP} 2 \mathrm{mg}$ $\mathrm{L}^{-1}$; B3= BAP $3 \mathrm{mg} \mathrm{L}^{-1}$.

\begin{tabular}{crcrrr}
\hline \multirow{2}{*}{$\begin{array}{c}\text { BAP } \\
\left(\mathrm{mg} \mathrm{L}^{-1}\right)\end{array}$} & $\begin{array}{c}\text { Núm. de brotes por } \\
\text { explante }\end{array}$ & $\begin{array}{c}\text { Altura de brote } \\
(\mathrm{cm})\end{array}$ & & $\begin{array}{c}\text { Núm. de brotes por } \\
\text { explante }\end{array}$ & $\begin{array}{c}\text { Altura de brote } \\
(\mathrm{cm})\end{array}$ \\
\hline B0=0 & $4.9 \pm 1.92 \mathrm{~b}$ & $2.2724 \pm 0.34 \mathrm{a}$ & & $3.1 \pm 1.5 \mathrm{~b}$ & $1.5387 \pm 0.39 \mathrm{a}$ \\
$\mathrm{B} 1=1$ & $26.65 \pm 7.74 \mathrm{a}$ & $1.1103 \pm 0.07 \mathrm{c}$ & & $29 \pm 7.9 \mathrm{a}$ & $1.1058 \pm 0.06 \mathrm{~b}$ \\
$\mathrm{~B} 2=2$ & $24.65 \pm 6.08 \mathrm{a}$ & $1.4971 \pm 0.08 \mathrm{~b}$ & & $31.5 \pm 15.82 \mathrm{a}$ & $0.7965 \pm 0.05 \mathrm{c}$ \\
$\mathrm{B} 3=3$ & $22.65 \pm 10.33 \mathrm{a}$ & $1.0796 \pm 0.07 \mathrm{c}$ & & $40.35 \pm 17.49 \mathrm{a}$ & $0.7083 \pm 0.03 \mathrm{~d}$ \\
\hline
\end{tabular}

Media con letras iguales no difieren estadísticamente (One-Way Anova, Tuckey, $p<0.05$ ).

El mayor número de brotes por explante fue de 40.35 en el segmento nodal a una concentración de $3 \mathrm{mg} \mathrm{L}^{-1}$, mientras el testigo presentó el menor número de brotes tanto en el segmento nodal como el apical con 4.9 y 3.1 respectivamente (Figura 1). Para la variable longitud de brote se observó diferencias significativas $(p<0.05)$ en las concentraciones de 6-BAP y en los dos tipos de explantes. 
a)

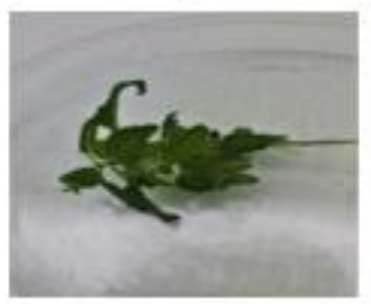

c)

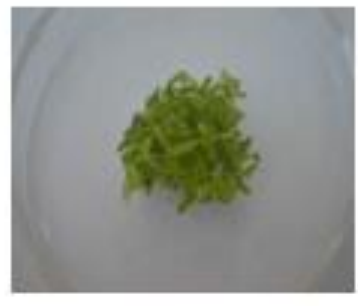

e)

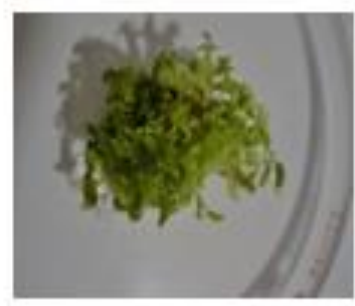

g)

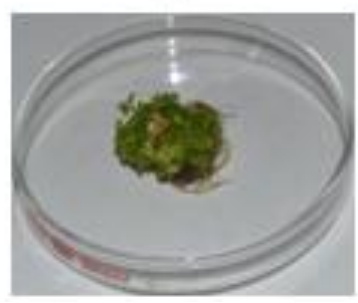

b)

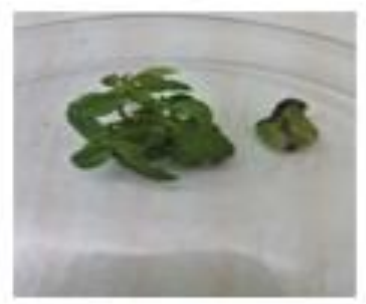

d)

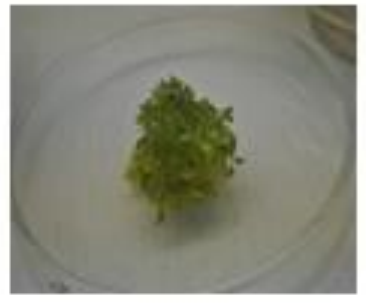

f)

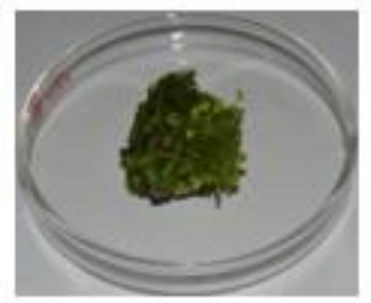

h)

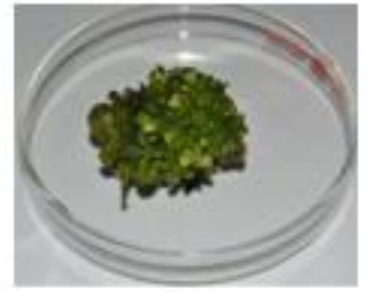

Figura 1. Resultados obtenidos al aplicar diferentes concentraciones de 6-BAP en la multiplicación de $L$. graveolens en dos tipos de explantes (segmentos nodales y apicales). B0 (testigo): segmento apical (a); segmento nodal (b). B1 (1 $\left.\mathrm{mg} \mathrm{L}^{-1}\right)$ : segmento apical (c); segmento nodal (d). B2 (2 mg L $\left.~^{-1}\right)$ : segmento apical (e); segmento nodal (f). B3 (3 mg L $\left.^{-1}\right)$ : segmento apical (g); y segmento nodal (h).

Los resultados muestran la influencia por tipo de explante y concentración de 6-BAP. El testigo del segmento apical presentó la mayor elongación con $2.27 \mathrm{~cm}$. En segmentos nodales se mostró la disminución de longitud de brote de manera gradual conforme aumentaba la concentración de 6-BAP, en segmentos apicales no se explica este patrón pues la concentración de $2 \mathrm{mg} \mathrm{L}^{-1}$ de 6BAP gener una longitud mayor a las otras dos concentraciones de 6-BAP (Figura 2). 


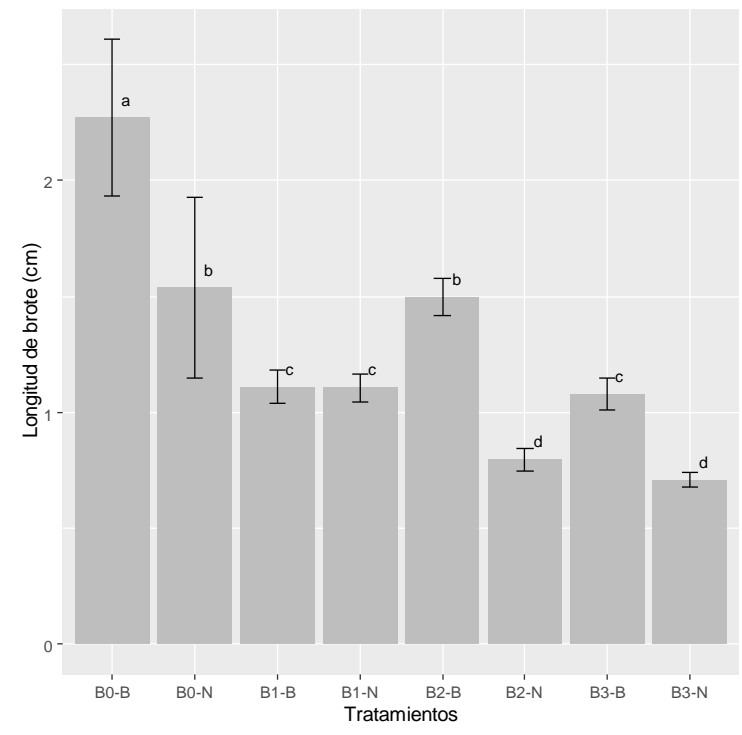

Figura 2. Efecto de diferentes concentraciones de 6-BAP en la longitud de brotes de $L$. graveolens en dos tipos de explantes. $\mathrm{B} 0-\mathrm{B}=$ testigo segmento apical; $\mathrm{B} 0-\mathrm{N}=$ testigo segmento nodal; $\mathrm{B} 1$ B= BAP $1 \mathrm{mg} \mathrm{L}^{-1}$ segmento apical; B1-N=BAP $1 \mathrm{mg} \mathrm{L}^{-1}$ segmento nodal; B2-B= BAP 2 mg $\mathrm{L}^{-1}$ segmento apical; B2-N= BAP $2 \mathrm{mg} \mathrm{L}^{-1}$ segmento nodal; B3-B $=B A P 3 \mathrm{mg} \mathrm{L}^{-1}$ segmento apical; B3-N= BAP $3 \mathrm{mg} \mathrm{L}^{-1}$ segmento nodal.

\section{Etapa III. Enraizamiento}

En todos los tratamientos se obtuvo $100 \%$ de enraizamiento, y no se presentaron diferencias significativas $(p<0.05)$ en el tamaño de la raíz entre tratamientos (Figura 3 ).

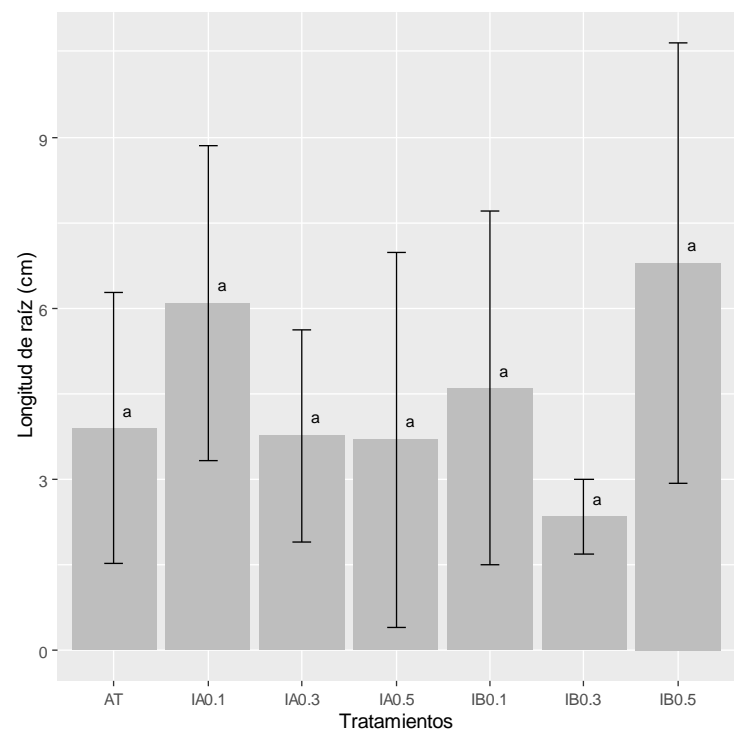

Figura 3. Efecto de diferentes concentraciones de AIA y AIB en la longitud de raíz de brotes de $L$. graveolens. AT = testigo; IA0.1 = AIA $0.1 \mathrm{mg} \mathrm{L}^{-1}$; IA0.3= AIA $0.3 \mathrm{mg} \mathrm{L}^{-1}$; IA0.5 = AIA $0.5 \mathrm{mg}$ $\mathrm{L}^{-1}$; IB0.1= AIB $0.1 \mathrm{mg} \mathrm{L}^{-1}$; IB0.3= AIBA $0.3 \mathrm{mg} \mathrm{L}^{-1}$; IB0.5= AIB $0.5 \mathrm{mg} \mathrm{L}^{-1}$. Media con letras iguales no difieren estadísticamente, Tukey $(p<0.05)$. 


\section{Etapa IV. Aclimatación}

Los resultados de aclimatación muestran que el incremento del fotoperiodo aumenta la sobrevivencia de las plántulas y este resultado se ve mejorado cuando se aplican micorrizas en combinación con el fotoperiodo, el mejor tratamiento (T5) obtuvo un porcentaje de sobrevivencia de $86.11 \%$ en comparación al testigo que solo tuvo $33.33 \%$ (Figura 4).

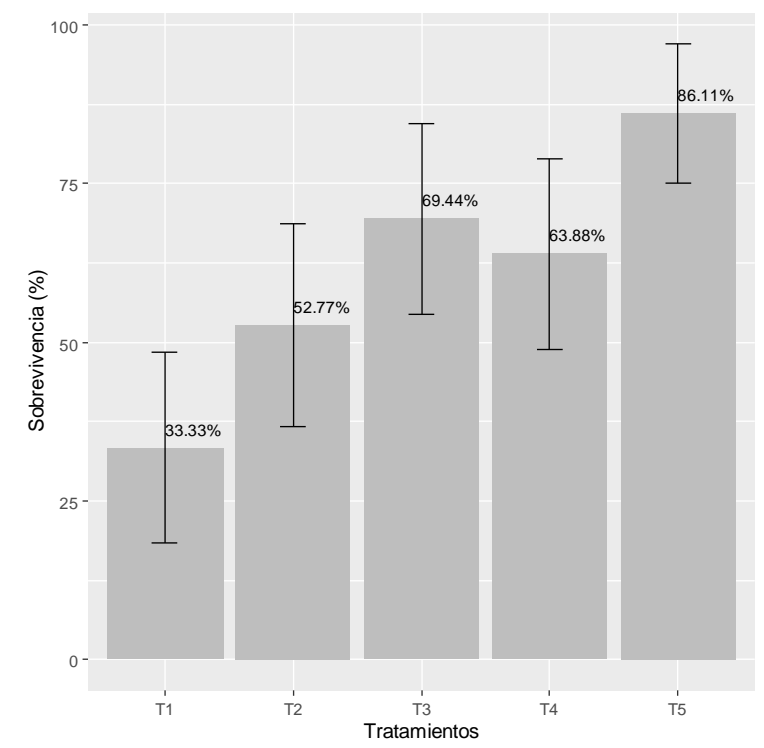

Figura 4 Porcentaje de superevivencia de $L$. graveolens en diferentes tratamientos con y sin micorrizas y dos tiempos de fotoperiodo. T1= testigo; $T 2=$ fotoperiodo de $16 \mathrm{~h} ; \mathrm{T} 3=$ fotoperiodo de $16 \mathrm{~h}$ más micorrizas; $\mathrm{T} 4=$ fotoperiodo de $24 \mathrm{~h}$; $\mathrm{T} 5=$ fotoperiodo de $24 \mathrm{~h}$ más micorrizas.

\section{Discusiones}

En la etapa de establecimiento del cultivo in vitro es requisito una adecuada técnica aséptica; sin embargo, otros factores a considerar son la fuente del explante, su estado fitosanitario y el tipo de explante. El método de desinfección empleado en este experimento durante el establecimiento in vitro ha sido utilizado en otras especies del género Lippia con diferentes resultados.

En este estudio el material vegetal fue obtenido de una plantación comercial y el porcentaje de explantes contaminados fue de $32.52 \%$, resultados similares fueron observados en L. gracilis donde obtuvieron $36.55 \%$ de explantes contaminados, también de material proveniente de campo, donde utilizaron el proceso de desinfección de etanol al $70 \%$ por un minuto y después realizaron la desinfección con hipoclorito de calcio al 3\% (Marinho et al., 2011).

Sin embargo, en la especie L. grata la contaminación no excedió el 3\% utilizando alcohol etílico al $70 \%$ por 1 minuto e hipoclorito de sodio al $2 \%$ con $50 \mu$ de Tween 20 durante 20 minutos con plantas provenientes de invernadero y con un tratamiento fitosanitario previo (Palhares-Neto et al., 2018). Como se menciona anteriormente la fuente del explante y la calidad fitosanitaria de la planta madre son decisivas para determinar buenos resultados. 
Por otro lado, el tipo de explante empleado puede presentar los riesgos mencionados por Cassells (2011) quien señala que el meristemo apical es el explante adecuado debido a que las hojas, los tallos y los explantes nodales tienen alto riesgo de contaminación dado que los microrganismos pueden alojarse en los espacios intercelulares e intracelulares, en la cutícula de las hojas y en cavidades en los tallos.

En el presente estudio la cantidad de explantes sanos obtenidos permitieron establecer la etapa de multiplicación empleando citocinina 6-BAP. Este tipo de regulador de crecimiento estimula la síntesis de proteínas y participan en el control del ciclo celular y aplicadas al medio de cultivo para inducción de brotes superan la dominancia apical y liberan a las yemas laterales de la latencia (Van Stadent et al., 2007) Las citocininas durante esta etapa, pueden ir solas o combinadas con una concentración menor de auxinas para generar una mejor respuesta morfogenética (Okubo et al., 1991; Sjahril et al., 2016).

En el caso de establecerse sin auxinas, se considera que hay aporte endógeno de auxinas que equilibran las necesidades hormonales para iniciar la organogénesis (Hu et al., 2017). Como en el caso de los explantes del cítrico Citrus sinensis y $C$. limon, donde la presencia de auxinas provoca la inhibición de formación de brotes adventicios haciendo necesaria su remoción (Hu et al., 2017).

En caléndula se menciona que la fuente de explante es una forma de tomar ventaja de la capacidad de sintetizar auxinas de explantes apicales, pues emplearon tres tipos de explantes nodales (apical, medio y basal) y fue en el explante apical donde se obtuvo mayor número de hojas y tamaño de brote adventicios, pero menor número de brotes, también el enraizamiento fue mayor en el explante apical y decreció en las regiones media y basal (Pimentel-Victorio et al., 2012).

En esta investigación la presencia de auxinas endógenas de los explantes (nudos y segmentos nodales) en combinación con tres concentraciones de 6-BAP generó un balance hormonal que estimuló la inducción de brotes axilares en la etapa de multiplicación. El tipo de explante no influyó significativamente en relación con el número de brotes obtenidos ni las concentraciones de 6-BAP afectaron significativamente los dos tipos de explantes.

Sin embargo, si hubo una diferencia con el testigo, donde se presentó el menor número de brotes en ambos explantes. Sin embargo, el número de brotes obtenidos con la hormona 6-BAP fue mayor a los resultados reportados en otras investigaciones de esta especie. Muñoz-Miranda et al. (2019) reporta un máximo de 6.4 brotes con BA a una concentración de $0.5 \mathrm{mg} \mathrm{L}^{-1}$, mientras CastellanosHernández et al. (2013) menciona 2.8 brotes por explante con BA a esa misma concentración.

El regulador del crecimiento 6-BAP también ha generado una respuesta diferente en la etapa de multiplicación en cada una de las especies del género Lippia donde ha sido empleado. En $L$. junelliana se obtuvieron 13.5 brotes por explante al añadir $0.04 \mu \mathrm{M}$ AIB más $4.4 \mu \mathrm{M}$ de BAP (Juliani et al. 1999) por otro lado, en $L$. alba el número de brotes obtenidos fue 6.4 brotes por explante con $5 \mathrm{mg} \mathrm{L}^{-1}$ de BAP (Gupta et al., 2001), en L. javanica se observaron 2.42 brotes por explante con $0.25 \mathrm{mg} \mathrm{L}^{-1}$ de BAP (Ara et al., 2010), igualmente en L. rotundifolia 2.6 brotes por explante con $0.33 \mu \mathrm{M}$ de BAP (Resende et al., 2015) y en L. integrifolia se obtuvo una media de 16 brotes por explante con $2.2 \mu \mathrm{M}$ BAP (Ianicelli et al., 2016), en cuanto a L. filifolia PereiraPeixoto et al. (2006) obtuvieron 27.03 brotes por explante a concentración de $4.5 \mu \mathrm{M}$ de BAP más $54 \mathrm{nM}$ de ANA. 
Esta diferencia entre segmentos apicales y nodales puede deberse a las auxinas endógenas producidas por el meristemo al igual que en los resultados reportados en caléndula (PimentelVictorio et al., 2012). En los resultados de segmentos nodales también se observó una disminución de la longitud de brote de $45 \%$ entre el testigo y la mayor concentración de la citocinina. El efecto fue directamente proporcional al incremento en la concentración de 6-BAP.

Este efecto del regulador fue reportado también por Juliani et al. (1999) en L. junelliana donde indican que la concentración de este regulador suprimió la elongación de los brotes. Por otro lado, Gupta et al. (2001) mencionan en la micropropagación de $L$. alba, que la concentración de bencilaminopurina afectó el crecimiento de los brotes producidos. De manera similar, PereiraPeixoto et al. (2006) en L. filifolia reportan que el número y el tamaño de brote se ajustó a una función cuadrática en respuesta a el incremento de este regulador.

Esto podría ser la causa de la disminución de la altura de los brotes cuando se aumenta la concentración de 6-BAP, ya que no solo limitarían la absorción de agua sino la de nutrientes. Análogamente, Resende et al. (2015) reportan en L. rotundifolia una disminución en altura de brote cuando la concentración de BAP fue mayor. Los resultados obtenidos en la etapa de enraizamiento no mostraron diferencias significativas para longitud de raíz entre el testigo y las concentraciones de AIA y AIB, también se observó $100 \%$ de enraizamiento en todos los tratamientos, este resultado ya había sido previamente reportado en otras especies del género Lippia, como en el caso de $L$. alba, donde se obtuvo en el testigo 100\% de formación de raíces (Gupta et al., 2001).

En L. junelliana el mejor porcentaje de enraizamiento se obtuvo en medio libre de hormonas que también produjo un sistema radicular largo y vigoroso (Juliani et al., 1999) Además en $L$. rotundifolia el testigo no supero a la mejor concentración de ANA $(0.44 \mu \mathrm{M})$ en formación de raíces y tamaño de brote, ya que fue similar a los obtenidos en las otras tres concentraciones empleadas (Resende et al., 2015) De igual forma, en L. filifolia el testigo obtuvo resultados semejantes a tres concentraciones de la auxina ANA pero tampoco superó a la mejor concentración de $0.11 \mu \mathrm{M}$ ANA en el número de raíces y $0.22 \mu \mathrm{M}$ en el largo de raíz (Pereira-Peixoto et al., 2006).

Los resultados obtenidos en la etapa de aclimatación muestran un efecto sinérgico entre el fotoperiodo y la biotización en la aclimatación de L. graveolens. Se ha observado que el fotoperiodo prolongado modifica la morfología y fisiología de las plantas al generar respuestas que favorecen el desarrollo de éstas. Adams y Langton (2005) mencionan que al aplicar un fotoperiodo prolongado, se observa un incremento en peso seco, y posiblemente un aumento de la fotosíntesis, además, se acompaña de una mayor área foliar con mayor número de células y tamaño celular.

Por otro lado, el fotoperiodo favorece un incremento de la estoma y la densidad estomática. Zacchini et al. (1997) mencionan que en cultivo in vitro de brotes de árboles frutales la densidad de las estomas fue mayor en hojas expuestas a luz continua, por otro lado, cuando se aplicó la misma cantidad de horas luz/oscuridad en diferentes intervalos $(4 / 2,16 / 8)$ se observó modificación en la densidad e índice estomático, siendo el resultado más favorable fue cuando se aplicó periodos cortos de luz/oscuridad.

Asimismo, en plántulas de fresa sometidas a fotoperiodos de 16 y $24 \mathrm{~h} \mathrm{luz}$, el crecimiento fue mayor que con luz solar, a pesar de que todos los tratamientos proporcionaron la misma luz diaria integral (Tsuruyama y Shibuya 2018). Mientras, Fortini et al. (2020) observaron también mayor 
influencia en el crecimiento y contenido de biomasa, con altas tasas fotosintéticas, afectando el metabolismo primario y secundario con diferencias en la producción de azúcares solubles, glucógeno, aminoácidos y compuestos bioactivos.

Asimismo, la biotización ha incrementado la supervivencia de las plantas cuando se aplica en la etapa de aclimatación. Mirjani et al. (2018) observaron en la etapa de aclimatación de Satureja khuzistanica al aplicar Glomus fasciculatum facilitaba la captación de diferentes elementos, contribuyendo a una rápida recuperación de las plantas durante esta etapa, además se observó un incremento de $17.4 \%$ en la supervivencia de éstas.

De igual forma, Bidabadi y Masoumian (2017) aplicaron Glomus intraradicens y evaluaron su efecto en plantas micropropagadas de Stevia rebaudiana, en condiciones de salinidad, observaron una disminución del daño al reducir del estrés oxidativo e incrementar la eficiencia fotosintética. Por otro lado, en plátano la aplicación de micorrizas disminuyó la mortalidad de plántulas micropropagadas e incremento calidad la calidad de éstas (Ortas et al., 2017), por lo tanto, puede deducirse que el fotoperiodo y la adición de micorrizasdurante la aclimatación, mejoran los porcentajes de supervivencia.

\section{Conclusiones}

Se logró establecer un protocolo de propagación in vitro para L. graveolens lo que permitirá la conservación de la especie a largo plazo, además del establecimiento de plantaciones especializadas homogéneas. La multiplicación de L. graveolens está influenciada por el tipo de explante, su aclimatación es dependiente de la luz que puede ser lograda con éxito con la aplicación de micorrizas para mejorar y reducir el estrés en esta etapa.

\section{Literatura citada}

Adams, S. R. and Langton, F. A. 2005. Photoperiod and plant growth: a review. J. Hortic. Sci. Biotechnol. 80(1):2-10.

Ara, N.; Safiul-Azam, F. M.; Lithy, S. S. and Rahmatullah, M. 2010. A study of plant growth hormones on in vitro clonal propagation of fever tea (Lippia javanica): a medicinal shrub. American-Eurasian J. Sustainable Agric. 4(3):274-279.

Ávila-Sosa, R.; Gastélum-Franco, M. G.; Camacho-Dávila, A.; Torres-Muñoz, J. V. and NevárezMoorillón G. V. 2010. Extracts of mexican oregano (Lippia berlandieri Schauer) with antioxidant and antimicrobial activity. Food Bio. Technol. 3(3):434-440.

Cassells, A. C. 2011. Detection and elimination of microbial endophytes and prevention of contamination in plant tissue culture. In plant tissue culture, development, and biotechnology. (Ed.). Trigiano, R. N. and Gray, D. J. CRC Press. Florida USA. 223$238 \mathrm{pp}$.

Cueto-Wong. M. C.; Rivas-Morales, C.; Alanís-Guzmán, M. G.; Oranday-Cárdenas, A.; AmayaGuerra, C. A.; Núñez-González, A.; Samaniego-Gaxiola, J. A. y Cano-Ríos, P. 2010. Antifungal properties of essential oil of mexican oregano (Lippia berlandieri) against Fusarium oxysporum f. sp. Lycopersici. Rev. Mex. Micol. 31(1):29-35. 
Dunford, N. T. and Vazquez, R. S. 2005. Effect of water stress on plant growth and thymol and carvacrol concentrations in Mexican oregano grown under controlled conditions J. Appl. Hortic. 7(1):20-22.

García-Pérez, E.; Castro-Álvarez, F. F.; Gutiérrez-Uribe, J. A. y García-Lara, S. 2012. Revisión de la producción, composición fitoquímica y propiedades nutracéuticas del orégano mexicano. Rev. Mex. Cienc. Agríc. 3(2):339-353.

Granados-Sánchez, D.; Martínez-Salvador, M.; López-Ríos, G. F. y Rodríguez-Yam, G. A. 2013. Ecología, aprovechamiento y comercialización del orégano (Lippia graveolens HBK) en mapimí, durango. Rev. Chapingo. Ser. Ciencias Forestales y del Ambiente. 19(2):305-322.

Gupta, S. K.; Khanuja, S. P. S. and Kumar, S. 2001. In vitro micropropagation of Lippia alba. Current Science-Bangalore. 81(2):206-209.

Hu, W.; Fagundez, S.; Katin, G. L.; Li, Y.; Li, W.; Chen, Y.; Wang, X.; Deng, Z.; Xie, S.; McAvoy, R. J. and Li, Y. 2017. Endogenous auxin and its manipulation influence in vitro shoot organogenesis of citrus epicotyl explants. Hortic. Res. 4(1):1-6.

Iannicelli, J; Perez-Torre, M.; Coviella, A.; Valle-Aguirre, E.; Elechosa, M. Á.; Van-Baren, C. M.; Pacheco, M. G. and Escandón, A. S. 2016. In vitro propagation of Lippia integrifolia (Griseb.) Hier. and detection of genetic instability through ISSR markers of in vitrocultured plants. Rev. Facult. Agrono. 115(1):67-76.

Juliani, H. R.; Koroch, A. R. and Trippi, V. S. 1999. Micropropagation of Lippia junelliana (Mold.) Tronc Plant Cell, Tissue Organ Culture. 59(3):175-179.

Kusters, K.; Achdiawan, R.; Belcher, B. and Pérez, M. R. 2006. Balancing development and conservation an assessment of livelihood and environmental outcomes of nontimber forest product trade in Asia, Africa, and Latin America. Ecol. Soc. 11(2):1-22.

Laferriere, J. E.; Weber, C. W. and Kohlhepp, E. A. 1991. Use and nutritional composition of some traditional mountain pima plan foods. J. Ethnobiology. 11(2):93-114.

Marinho, M. J.; Albuquerque, C. C.; Morais, M. B.; Souza, M. C. y Silva, K. M. 2011. Estabelecimento de protocolo para micropropagação de Lippia gracilis Schauer. Rev. Brasileira Plantas Medicinais. 13(2):246-252.

Marques, P. A. A.; Bernardi-Filho, L. and Oliveira, R. B. 2009. Oregano production under various water depths estimated by means of the class A pan evaporation. Horticultura Brasileira. 27(1):59-63.

Martínez-Rocha, A.; Puga, R.; Hernández-Sandoval, L.; Loarca-Piña, G. and Mendoza, S. 2008. Antioxidant and antimutagenic activities of mexican oregano (Lippia graveolens Kunth) Plant Foods Human Nutrition. 63(1):1-5.

Martínez-Velázquez, M.; Rosario-Cruz, R.; Castillo-Herrera, G.; Flores-Fernández, J. M.; Álvarez, A. H. and Lugo-Cervantes, E. 2011. Acaricidal effect of essential oils from Lippia graveolens (Lamiales: Verbenaceae), Rosmarinus officinalis (Lamiales: Lamiaceae), and Allium sativum (Liliales: Liliaceae) against Rhipicephalus (Boophilus) microplus (Acari: Ixodidae) J. Medical Entomol. 48(4):822-827.

Muñoz-Miranda, L. A.; Rodríguez-Sahagún, A.; Acevedo Hernández, G. J.; Cruz-Martínez, V. O.; Torres-Morán, M. I.; Lépiz-Ildefonso, R.; Aarland, R. C. and Castellanos-Hernández, O. A. 2019. Evaluation of Somaclonal and ethyl methane sulfonate-induced genetic variation of mexican oregano (Lippia graveolens HBK). Agronomy. 9(4):166-176.

Murashige, T. and Skoog, F. 1962. A revised médium for rapid growth and bioassays with tobacco. Tissue Cultures Physiologia Plantarum. 15(3):473-497. 
Ocampo-Velázquez, R. V.; Malda-Barrera, G. X. y Suárez-Ramos, G. 2009. Biología reproductiva del orégano mexicano (Lippia graveolens Kunth) en tres condiciones de aprovechamiento. Agrociencia. 43(5):475-482.

Okubo, H.; Wada, K. and Uemoto, S. 1991. In vitro morphogenetic response and distribution of endogenous plant hormones in hypocotyl segments of snapdragon (Antirrhinum majus L.). Plant Cell Reports. 10(10):501-504.

Palhares-Neto, L.; Souza, L. M.; Bizerra, M. M.; Cavalcanti, M. C.; Rangel, C. T. and Ulisses, C. 2018. Controlling hyperhydricity in micropropagated plants of Lippia grata Schauer (Verbenaceae), a native species of a dry seasonal tropical forest with pharmacological potential. Braz. J. Bot. 41(3):529-538.

Paredes-Aguilar, M. D. L. C.; Gastélum-Franco, M. G.; Silva-Vázquez, R. y NevárezMoorillón, G. V. 2007. Efecto antimicrobiano del orégano mexicano (Lippia berlandieri Schauer) y de su aceite esencial sobre cinco especies del género vibrio. Rev. Fito. Mex. 30(3):261-267.

Pereira-Peixoto, H. P.; Salimena, F. R. G.; Oliveira, S. M.; Silva, G. L.; Oliveira-Pierre P. M.; Viccin, I. L. F. and Otoni, W. C. 2006. In vitro propagation of endangered Lippia filifolia Mart. and Schauer ex Schauer. In vitro Cellular Developmental Biology-Plant. 42(6):558-561.

Pimentel-Victório, C.; Salgueiro-Lage, C. L. and Sato, A. 2012. Tissue culture techniques in the proliferation of shoots and roots of Calendula officinalis. Rev. Cienc. Agron. 43(3):539-545.

Resende, C. F. D.; Bianchetti, R. E.; Oliveira, A. M. S. D.; Braga, V. F. and Peixoto, P. H. P. 2015. In vitro propagation and acclimatization of Lippia rotundifolia, an endemic species of brazilian campos Rupestres. Rev. Ciênc. Agron. 46(3):582-589.

Sjahril, R.; Haring, F.; Riadi, M.; Rahim, M. D.; Khan, R. S.; Amir, A. and Trisnawaty, A. 2016. Performance of NAA, 2iP, BAP and TDZ on callus multiplication, shoots initiation and growth for efficient plant regeneration system in Chrysanthemum (Chrysanthemum morifolium Ramat.). Inter. J. Agric. System. 4(1):52-61.

Soto, R. J.; Hernández, A. F.; Franco, R. C. y Silva, R. 2007. Identificación y selección de genotipos de orégano (Lippia berlandieri Schauer) sobresalientes en producción de timol y carvacrol. Rev. Chapingo Ser. Zonas Áridas. 6(1):25-36.

Tsuruyama, J. and Shibuya, T. 2018. Growth and flowering responses of seed-propagated strawberry seedlings to different photoperiods in controlled environment chambers. HortTechnology. 28(4):453-458.

Villavicencio-Gutiérrez, E.; Cano-Pineda, A. y García-Cuevas, X. 2010. Metodología para determinar las existencias de orégano (Lippia graveolens HBK) en rodales naturales de Parras de la Fuente Coahuila. Folleto técnico núm. 42.42 p.

Zacchini, M.; Morini, S. and Vitagliano, C. 1997. Effect of photoperiod on some stomatal characteristics of in vitro cultured fruit tree shoots. Plant Cell, Tissue Organ Culture. 49(3):195-200. 